\title{
Study on Molecular Mechanism of Yiqing Capsule in Treating Upper Respiratory Tract Infection Based on Network Pharmacology
}

\author{
Yanwu Zhao, Junhui Hu* \\ Department of Pharmacy, The Affiliated Hospital of Chengde Medical College, Chengde, China \\ Email: ^hujhcyfy@163.com
}

How to cite this paper: Zhao, Y.W. and Hu, J.H. (2020) Study on Molecular Mechanism of Yiqing Capsule in Treating Upper Respiratory Tract Infection Based on Network Pharmacology. Chinese Medicine, 11, 1-8.

https://doi.org/10.4236/cm.2020.111001

Received: February 2, 2020

Accepted: March 28, 2020

Published: March 31, 2020

Copyright () 2020 by author(s) and Scientific Research Publishing Inc. This work is licensed under the Creative Commons Attribution International License (CC BY 4.0).

http://creativecommons.org/licenses/by/4.0/ (c) (i) Open Access

\begin{abstract}
Objective: To investigate the possible mechanism of Yiqing Capsules in the treatment of upper respiratory tract infection based on network pharmacology. Methods: The main active components of Yiqing Capsules were selected on TCMSP database; the targets of upper respiratory tract infection were selected on GeneCards database. The drug-compound-target network and PPi network were constructed through STRING database and soft Cytoscape 3.7.2. Soft $\mathrm{R}$ was used to perform GO enrichment analysis and KEGG pathway enrichment analysis of main targets. Results: According to the screening conditions, 48 active compounds and 171 related targets were obtained. GO enrichment analysis obtained 2333 items, KEGG pathway enrichment analysis obtained 2248 items, including Kaposi sarcoma-associated herpesvirus infection, Human cytomegalovirus infection, Epstein-Barr virus infection, PI3K-Akt signaling pathway, etc. Conclusion: Yiqing capsules play a therapeutic role in upper respiratory tract infection through multi-target and multi-pathway.
\end{abstract}

\section{Keywords}

Yiqing Capsule, Upper Respiratory Tract Infection, Network Pharmacology

\section{Introduction}

Upper respiratory infection is a general term that includes acute inflammation of the nasal cavity, pharynx, or larynx. Generalized sense is not a diagnosis of a disease, but a group of diseases, including the common cold, viral pharyngitis, laryngitis, herpetic pharyngitis, eustachian conjunctiva fever, and bacterial pha- 
ryngo-tonsillitis. At present, there is no specific drug treatment for upper respiratory tract infection, but antibiotics and antiviral drugs are used according to the relevant examination results. Research shows that traditional Chinese medicine (TCM) with heat-clearing, detoxification and antiviral effects can also be selected, which helps to improve symptoms and shorten the course of disease. Yiqing Capsule is a Chinese herbal medicine compound preparation which is composed of Scutellaria baicalensis, Coptis chinensis, and Rheum officinale. The capsule has functions of heat-clearing and detoxicating, removing blood stasis, hemostasis, etc. It is suitable for the treatment of body heat irritability, eye red sore of mouth, sore throat and gingival swelling pain, and constipation [1]. The capsule is used for clinical treatment on acute and chronic pharyngitis [2] [3] [4], acne [5], periodontitis [6], etc. It is also reported that Yiqing Capsule has good therapeutic effect in the acute stage of upper respiratory tract infection [7]. However, the relevant molecular mechanisms are unclear and unreported. Therefore, our study explored and predicted the relevant targets and molecular mechanisms of Yiqing Capsule in the treatment of upper respiratory tract infection based on network pharmacology, in order to provide theoretical data for clinical application.

\section{Materials and Methods}

\subsection{Selected the Compounds and Targets of Yiqing Capsule}

Searched chemical components of Scutellaria baicalensis, Coptis chinensis, Rheum officinale through Traditional Chinese Medicine Systems Pharmacology Database and Analysis Platform (TCMSP) database, used oral bioavailability $(\mathrm{OB}) \geq 30 \%$ and drug-likeness $(\mathrm{DL}) \geq 0.18$ as screening conditions to get the relevant compounds and targets.

\subsection{Constructed the Drug-Compound-Target Network and PPi Network}

Use "upper respiratory tract infection" as a keyword to screen the target genes on GeneCards database (www.genecards.org). Then make comparison with the target genes of the main chemical components of Yiqing Capsule, screened out the same target genes. Then construct drug-compound-target network and PPi network through soft Cytoscape 3.7.2 and STRING database.

\subsection{Target Pathway Analysis}

The key genes to the main chemical components of Yiqing Capsules in treating upper respiratory tract infection were used for Gene Ontology (GO) enrichment analysis and Kyoto Encyclopedia of Genes and Genomes (KEGG) enrichment analysis. GO enrichment analysis included biological process (BP), molecular function (MF), cellular component (CC). Then screen the gene-pathway by KEGG enrichment analysis. All analysis used soft R, “org.Hs.eg.db”, “clusterProfiler", "enrichplot" and "ggplot2", p-value $<0.5$. 


\section{Results}

\subsection{Compounds and Targets of Yiqing Capsule}

Searched chemical components of Coptis chinensis, Radix Scutellariae and Rhubarb through TCMSP database, after eliminated the same compounds, we selected out 48 active compounds, 30 for Scutellaria baicalensis, 11 for Coptis chinensis, 7 for Rheum officinale, the results are shown in Table 1. Search 6391 target genes on GeneCards database with the keyword "upper respiratory tract infection". 171 target genes of Yiqing Capsule in treating upper respiratory tract infection were obtained through matching, the results are shown in Figure 1.

Table 1. Basic information of active components in Yiqing Capsule.

\begin{tabular}{|c|c|c|c|c|c|}
\hline NO & Mol ID & components & MW & $\mathrm{OB}(\%)$ & $\mathrm{DL}$ \\
\hline \multicolumn{6}{|c|}{ Scutellaria baicalensis (30 components) } \\
\hline 1 & MOL000073 & ent-Epicatechin & 290.29 & 48.96 & 0.24 \\
\hline 2 & MOL000173 & wogonin & 284.28 & 30.68 & 0.23 \\
\hline 3 & MOL000228 & (2R)-7-hydroxy-5-methoxy-2-phenylchroman-4-one & 270.3 & 55.23 & 0.2 \\
\hline 4 & MOL000358 & beta-sitosterol & 414.79 & 36.91 & 0.75 \\
\hline 5 & MOL000359 & sitosterol & 414.79 & 36.91 & 0.75 \\
\hline 6 & MOL000449 & Stigmasterol & 412.77 & 43.83 & 0.76 \\
\hline 7 & MOL000525 & Norwogonin & 270.25 & 39.4 & 0.21 \\
\hline 8 & MOL000552 & 5,2'-Dihydroxy-6,7,8-trimethoxyflavone & 344.34 & 31.71 & 0.35 \\
\hline 9 & MOL001458 & coptisine & 320.34 & 30.67 & 0.86 \\
\hline 10 & MOL001490 & bis $[(2 S)$-2-ethylhexyl] benzene-1,2-dicarboxylate & 390.62 & 43.59 & 0.35 \\
\hline 11 & MOL002714 & baicalein & 270.25 & 33.52 & 0.21 \\
\hline 12 & MOL002879 & Diop & 390.62 & 43.59 & 0.39 \\
\hline 13 & MOL002897 & epiberberine & 336.39 & 43.09 & 0.78 \\
\hline 14 & MOL002909 & 5,7,2,5-tetrahydroxy-8,6-dimethoxyflavone & 376.34 & 33.82 & 0.45 \\
\hline 15 & MOL002910 & Carthamidin & 288.27 & 41.15 & 0.24 \\
\hline 16 & MOL002913 & Dihydrobaicalin_qt & 272.27 & 40.04 & 0.21 \\
\hline 17 & MOL002914 & Eriodyctiol (flavanone) & 288.27 & 41.35 & 0.24 \\
\hline 18 & MOL002915 & Salvigenin & 328.34 & 49.07 & 0.33 \\
\hline 19 & MOL002917 & 5,2',6'-Trihydroxy-7,8-dimethoxyflavone & 330.31 & 45.05 & 0.33 \\
\hline 20 & MOL002925 & 5,7,2',6'-Tetrahydroxyflavone & 286.25 & 37.01 & 0.24 \\
\hline 21 & MOL002927 & Skullcapflavone II & 374.37 & 69.51 & 0.44 \\
\hline 22 & MOL002928 & oroxylin a & 284.28 & 41.37 & 0.23 \\
\hline 23 & MOL002932 & Panicolin & 314.31 & 76.26 & 0.29 \\
\hline 24 & MOL002934 & NEOBAICALEIN & 374.37 & 104.34 & 0.44 \\
\hline 25 & MOL002937 & DIHYDROOROXYLIN & 286.3 & 66.06 & 0.23 \\
\hline
\end{tabular}




\section{Continued}

\begin{tabular}{|c|c|c|c|c|c|}
\hline 26 & MOL008206 & Moslosooflavone & 298.31 & 44.09 & 0.25 \\
\hline 27 & MOL010415 & 11,13-Eicosadienoic acid, methyl ester & 322.59 & 39.28 & 0.23 \\
\hline 28 & MOL012245 & 5,7,4'-trihydroxy-6-methoxyflavanone & 302.3 & 36.63 & 0.27 \\
\hline 29 & MOL012246 & 5,7,4'-trihydroxy-8-methoxyflavanone & 302.3 & 74.24 & 0.26 \\
\hline 30 & MOL012266 & rivularin & 344.34 & 37.94 & 0.37 \\
\hline \multicolumn{6}{|c|}{ Coptis chinensis (11 components) } \\
\hline 31 & MOL000098 & quercetin & 302.25 & 46.43 & 0.28 \\
\hline 32 & MOL000622 & Magnograndiolide & 266.37 & 63.71 & 0.19 \\
\hline 33 & MOL000785 & palmatine & 352.44 & 64.6 & 0.65 \\
\hline 34 & MOL001454 & berberine & 336.39 & 36.86 & 0.78 \\
\hline 35 & MOL001458 & coptisine & 320.34 & 30.67 & 0.86 \\
\hline 36 & MOL002668 & Worenine & 334.37 & 45.83 & 0.87 \\
\hline 37 & MOL002894 & berberrubine & 322.36 & 35.74 & 0.73 \\
\hline 38 & MOL002897 & epiberberine & 336.39 & 43.09 & 0.78 \\
\hline 39 & MOL002903 & (R)-Canadine & 339.42 & 55.37 & 0.77 \\
\hline 40 & MOL002904 & Berlambine & 351.38 & 36.68 & 0.82 \\
\hline 41 & MOL002907 & Corchoroside A_qt & 404.55 & 104.95 & 0.78 \\
\hline \multicolumn{6}{|c|}{ Rheum officinale (7 components) } \\
\hline 42 & MOL000096 & $(-)$-catechin & 290.29 & 49.68 & 0.24 \\
\hline 43 & MOL000358 & beta-sitosterol & 414.79 & 36.91 & 0.75 \\
\hline 44 & MOL000471 & aloe-emodin & 270.25 & 83.38 & 0.24 \\
\hline 45 & MOL002235 & EUPATIN & 360.34 & 50.8 & 0.41 \\
\hline 46 & MOL002268 & rhein & 284.23 & 47.07 & 0.28 \\
\hline 47 & MOL002281 & Toralactone & 272.27 & 46.46 & 0.24 \\
\hline 48 & MOL002297 & Daucosterol_qt & 386.73 & 35.89 & 0.7 \\
\hline
\end{tabular}

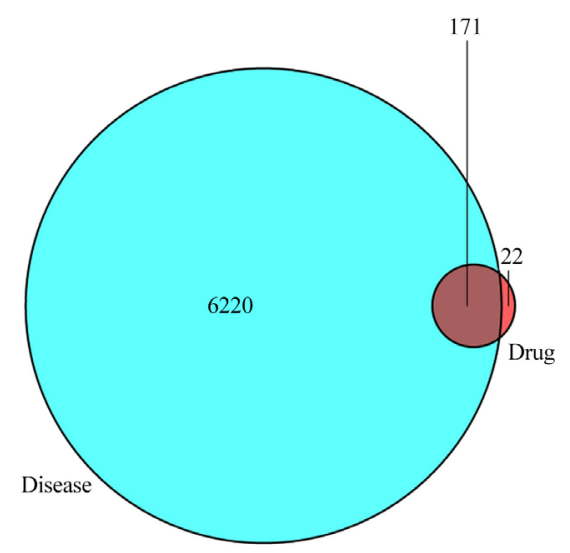

Figure 1. Venny figure for Yiqing Capsule in treating upper respiratory tract infection. (The aquamarine blue circle shows 6391 target genes of upper respiratory tract infection, the red circle shows 193 targets in Yiqing Capsule. The overlap represents a total of 171 genes in Yiqing Capsule in treating the disease.) 


\subsection{Constructed the Drug-Compound-Target Network and PPi Network}

We constructed the drug-compound-target network of Yiqing Capsule in treating upper respiratory tract infection on soft Cytoscape, at last we got 227 nodes and 798 edges, as is shown in Figure 2. PPi network was constructed through STRING database, the result is shown in Figure 3.

\subsection{Target Pathway Analysis}

Our research got $2333 \mathrm{GO}$ items of 48 main targets, with $68 \mathrm{CC}$ items, $2117 \mathrm{BP}$ items and $148 \mathrm{MF}$ items, such as nuclear receptor activity, transcription factor activity, direct ligand regulated sequence-specific DNA binding and cytokine receptor binding, etc. The top 20 items are shown in Figure 4. We also got 2248 gene-pathway by KEGG enrichment analysis, such as Hepatitis B, IL17 signaling pathway, Kaposi sarcoma-associated herpesvirus infection, Human cytomegalovirus infection, Epstein-Barr virus infection, PI3K-Akt signaling pathway, etc, the results are shown in Figure 5. In Figure 4 and Figure 5, the colour of column nodes shows the $\mathrm{P}$ value, from blue to red indicates that $\mathrm{P}$ value is smaller, the gradual change of column shape from long to short indicates that there are more enrichment targets.

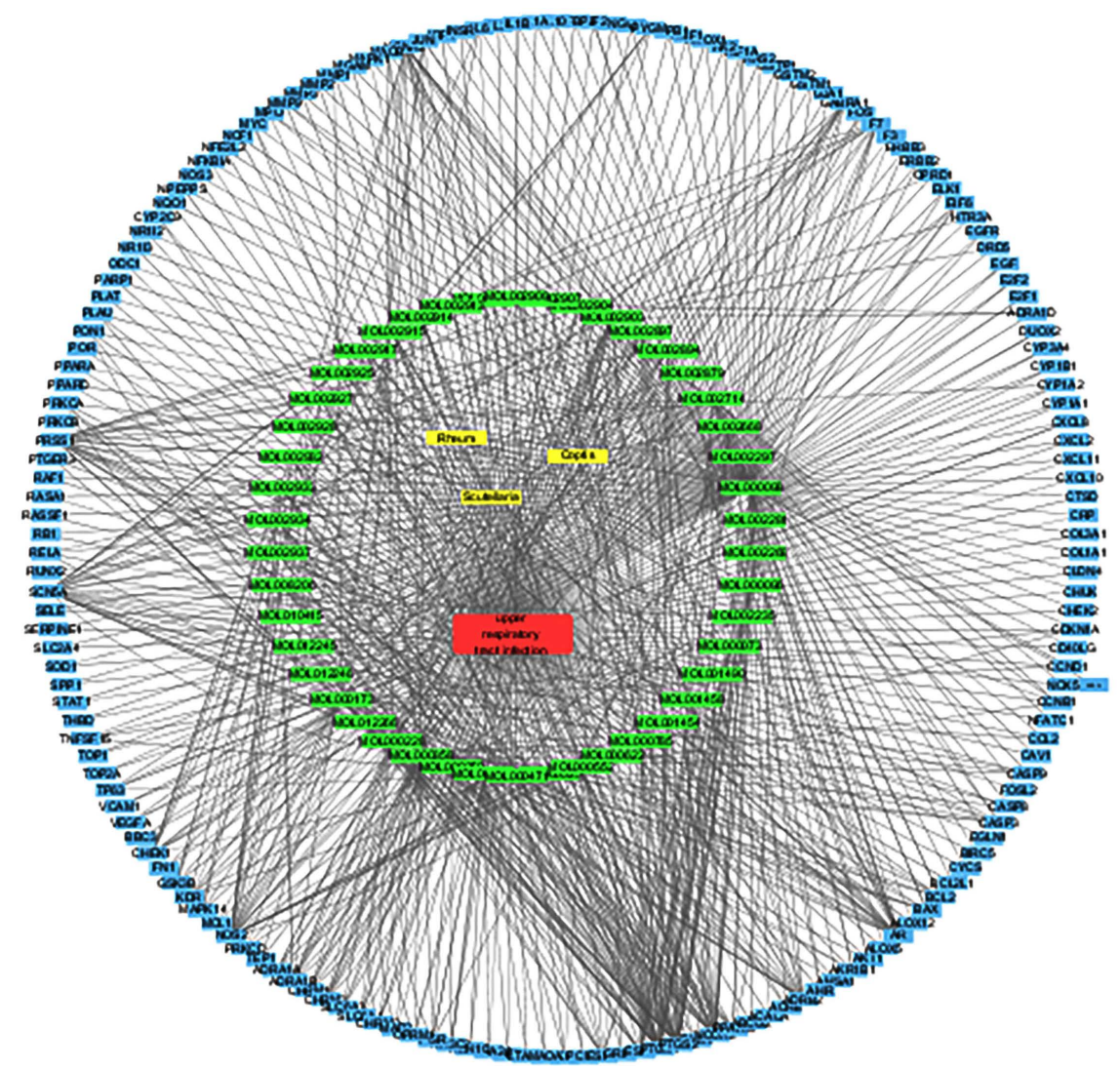

Figure 2. Compound-target-pathway network of Yiqing Capsule in treating upper respiratory tract infection. (The red is desease, the yellow is Chinese medical herbs of Yiqin Capsule, the green is main compounds, the blue is gene targets.) 


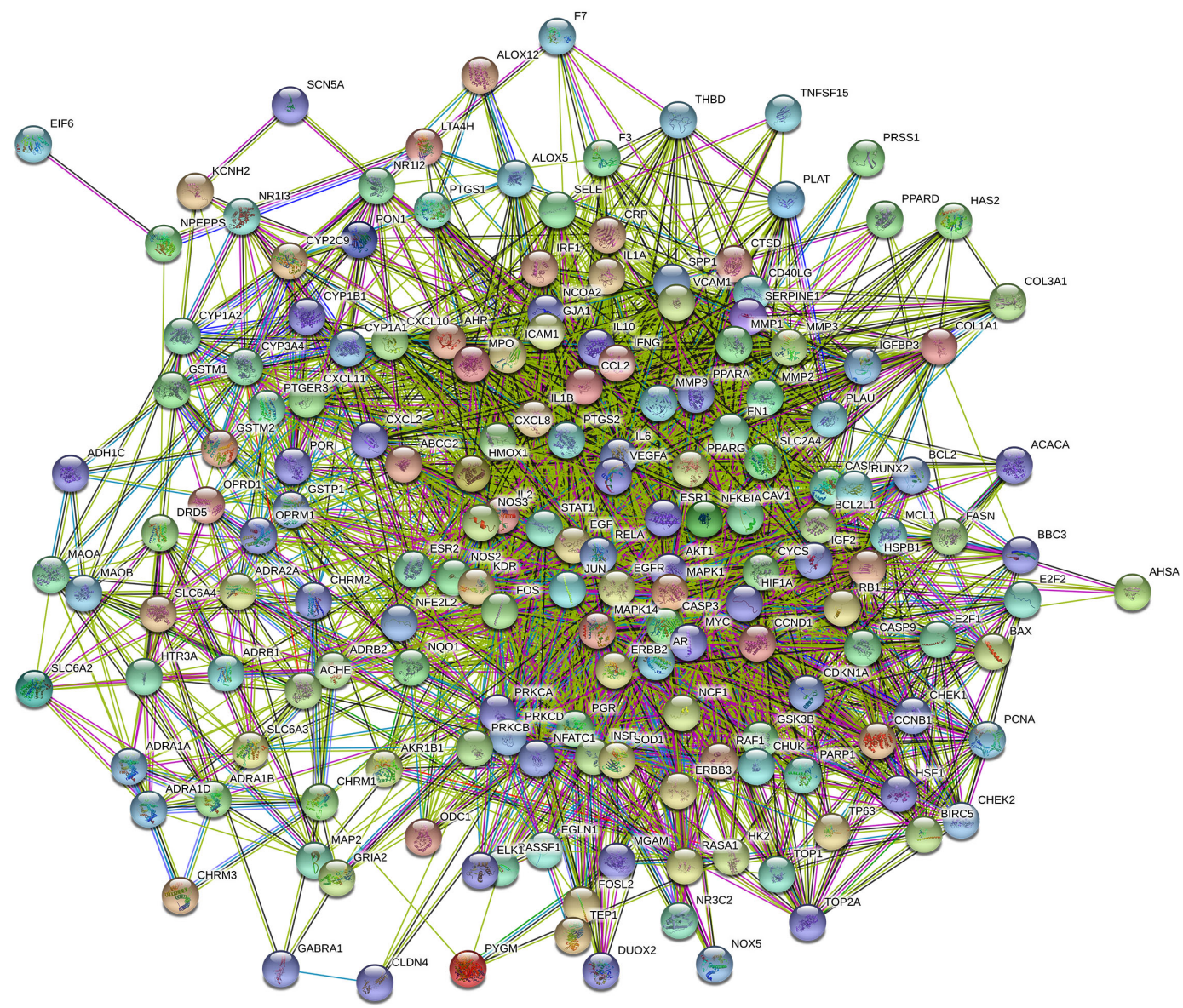

Figure 3. PPi network of Yiqing Capsule.

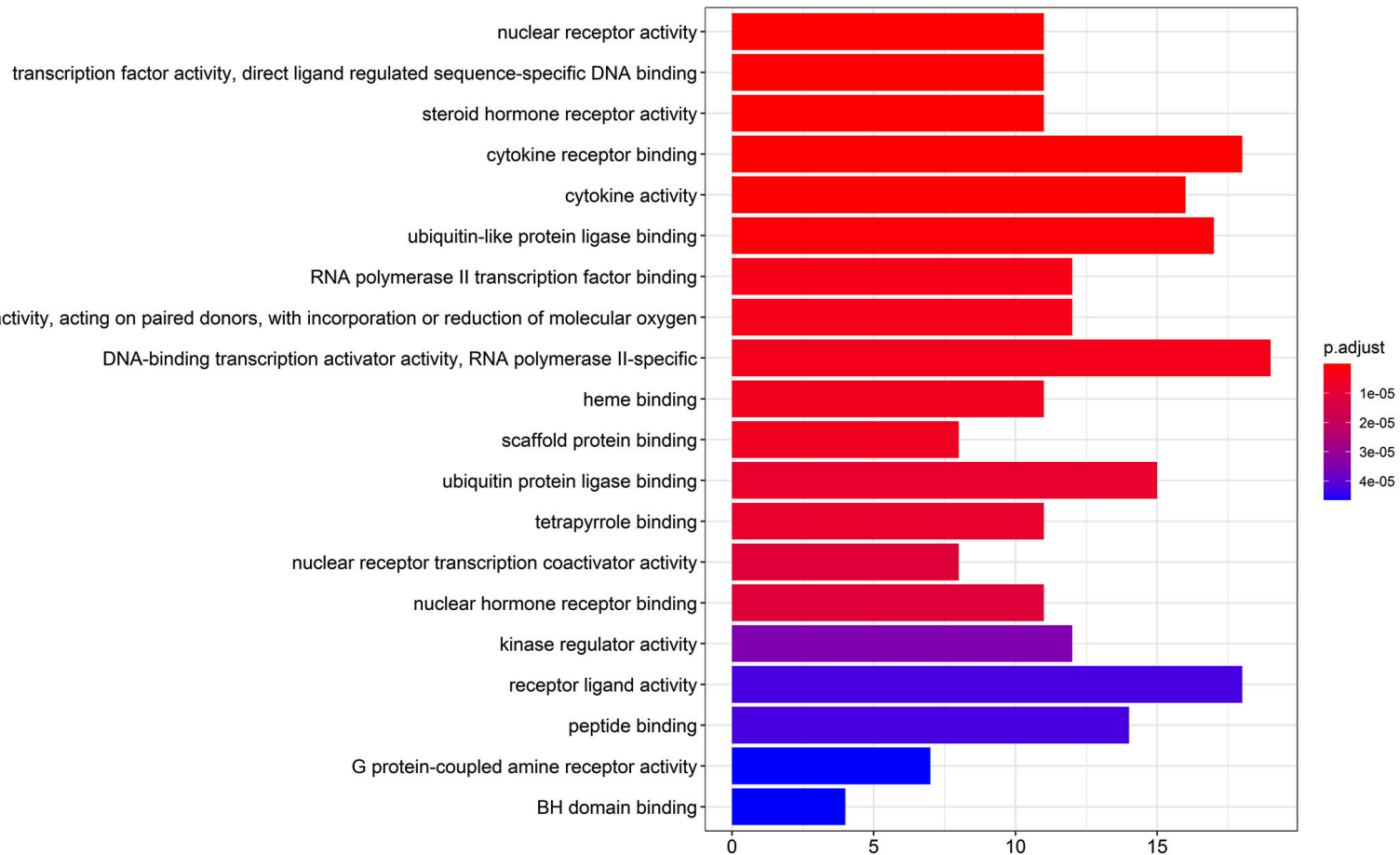

Figure 4. GO enrichment analysis of targets of Yiqing Capsule. 


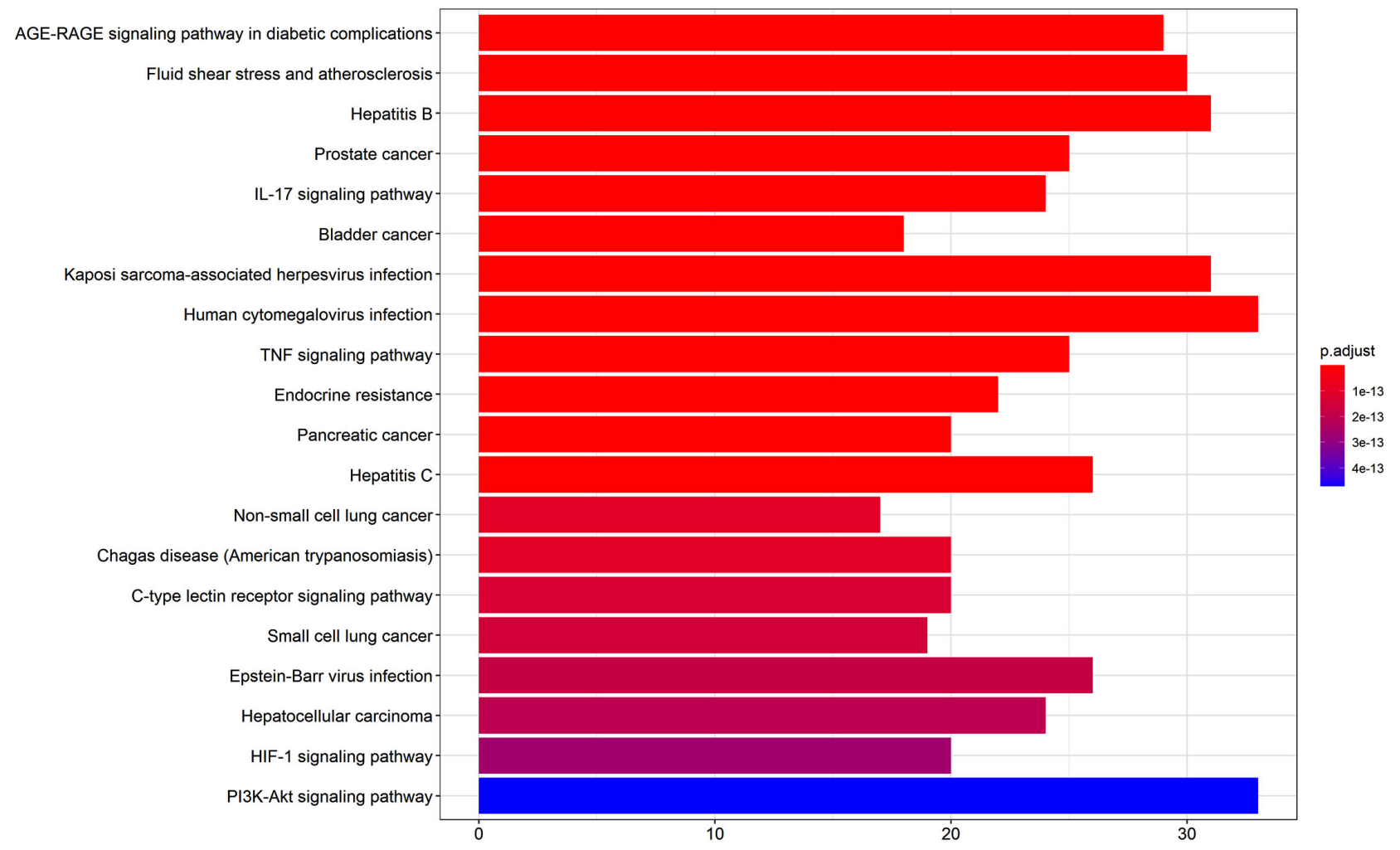

Figure 5. KEGG enrichment analysis of targets of Yiqing Capsule.

\section{Discussion}

Network pharmacology is a method to study the network relationship among drugs, diseases and targets, which has been widely used in pharmacology research, and is helpful to quickly find the targets and molecular mechanisms of drugs. Our study analyzed the main active compounds and related targets of Yiqing Capsule in the treatment of upper respiratory tract infection through network pharmacology. The result is shown that Yiqing Capsule plays an important role in the treatment of upper respiratory tract infection through multiple compounds, multiple targets and multiple pathways. Liu Wei found that the serum level of il-17 in children with recurrent upper respiratory tract infection was significantly higher than that in healthy children. The result of his study showed that il-17 was an independent factor in the occurrence of children with upper respiratory tract infection by Cox statistical analysis [8]. In our study, we found that the active component pathway of Yiqing Capsule includes il-17 signaling pathway. Therefore, combined with the characteristics of il-17 expression level in the above clinical reports, certain theoretical basis can be provided for the treatment of upper respiratory tract infection by Yiqing Capsule.

Our study also explored the potential components, targets and signaling pathways of Yiqing Capsule in the treatment of upper respiratory tract infection by network pharmacology, providing a theoretical basis for the study of molecular signaling pathways and clinical applications. However, the complex relationship between Yiqing Capsule and diseases still needs further clinical verification. 


\section{Funding}

This study was funded by the Study and Development Fund for Sciences and Technology in Chengde City (No. 201701A087).

\section{Conflicts of Interest}

The authors declare no conflicts of interest regarding the publication of this paper.

\section{References}

[1] Ding, H., Yan, B., Tian, L., et al. (2011) A Mutli-Center Randomized Double-Blind Controlled Trial on the Function of Yiqing Capsule Treat. Liaoning Journal of Traditional Chinese Medicine, 38, 1468-1490.

[2] Liu, X. (2017) Study on the Effect of Chronic Pharyngitis Mixture on Chronic Pharyngitis. Medical Innovation of China, 14, 112-114.

[3] Zhang, Y. and Wang, Y. (2016) Observation of Yiqing Capsule Combined with Antibiotic in the Treatment of 118 Cases of Acute Pharyngitis. Nei Mongol Journal of Traditional Chinese Medicine, 2, 74-75.

[4] Zhang, L. (2018) Analysis of the Efficacy of Yiqing Capsule Combined with Meifeng in Treating Chronic Pharyngitis. Clinical Research, 26, 118-120.

[5] Jiang, R. and Zhang, C. (2010) Clinical Observat Ion on 72 Cases of Acne Vulgaris Treated by Yiqing Capsule. Journal of Traditional Chinese Medicine, 51, 996-998.

[6] Liu, C., Xie, B., Cai, L., et al. (2018) Clinical Study on Yiqing Capsules Combined with Minocycline in Treatment of Chronic Periodontitis. Drugs \& Clinic, 33, 2083-2086.

[7] Hou, Y., Liu, X. and Xu, Q. (2001) Clinical Observation of 156 Cases of Acute Upper Respiratory Tract Infection Treated by Yiqing Capsule and Antibiotics. West China Journal of Pharmaceutical Sciences, 16, 401.

[8] Liu, W. (2018) Levels and Clinical Significances of Serum 25-Hydroxyvitamin D3, IL-10 and IL-17 in Children with Recurrent Upper Respiratory Infection. China Medical Herald, 15, 80-83. 